\title{
Implications of Dissipativity on Stability of Economic Model Predictive Control - The Indefinite Linear Quadratic Case
}

\author{
Olumuyiwa I. Olanrewaju*, Jan M. Maciejowski \\ Department of Engineering, University of Cambridge, Trumpington St, Cambridge \\ CB2-1PZ, UK
}

\begin{abstract}
In contrast to the conventional model predictive control (MPC) approach to control of a given system where a positive-definite objective function is employed, economic MPC employs a generic cost which is related to the 'economics' of the process as the objective function in the regulation layer. Often, stability proofs of the closed-loop system are based on strict-dissipativity of the system with respect to this objective function. In this paper, we focus on linear systems with indefinite quadratic costs. We show that while strict-dissipativity guarantees stability of the closed-loop system, it is not required. Hence we formulate a necessary and sufficient condition that guarantees asymptotic stability of the closed loop system. This condition comes down to the existence of two distinct storage functions for which the system is dissipative.
\end{abstract}

Keywords: Economic Model Predictive Control, Dissipativity, Strict-dissipativity, Stability

\section{Introduction}

In conventional model predictive control (MPC) and linear quadratic regulator (LQR) approaches to the control of a given system, the optimal steady-state of the system with respect to the 'economic' cost is first computed then deviations from this optimal steady-state are minimized using a dynamic regulation layer. This dynamic regulation layer is usually referred to as the advanced process control layer (which uses MPC or LQR). The cost function employed in the MPC plays a very important role for the stability of the closed-loop system. It has been established in both the MPC and LQR literature that under nominal

\footnotetext{
*Corresponding author. Tel.: +44 1223748357

Email addresses: oio24@cam.ac.uk (Olumuyiwa I. Olanrewaju), jmm@eng.cam.ac.uk (Jan M. Maciejowski)
} 
operation, stability of the closed loop system (using MPC or LQR) can be guaranteed provided the system is stabilizable, the cost function is positive-definite and a suitable terminal cost is used [1-3].

Economic MPC (e-MPC) employs a different approach to predictive control. The 'economic' cost is used directly in the dynamic regulation layer. Since this cost is generic and not guaranteed to be positive-definite as in conventional MPC, proof of stability can not be based on this property of the cost function. Strict-dissipativity, a property of the system with respect to the cost function, has often been used to overcome this limitation. This strict-dissipativity condition plays a central role in the analysis of economic MPC. The sufficiency of strict-dissipativity condition for optimality of steady-state operation was established in [4-6] while $[4,7]$ further showed that this same strict-dissipativity condition guarantees stability of the closed-loop system obtained using the economic cost in the dynamic regulation layer. Thus, optimality of steady-state operation and stability of the dynamic regulation to this steady-state are both guaranteed by the same strict-dissipativity condition. It has also been proven that a less strict condition, dissipativity, also guarantees optimality of steadystate operation and is close to being necessary for steady state operation to be optimal (under some additional controllability assumption)[5,6]. Simulations however show that in some cases, dissipativity (and not strict-dissipativity) appears to be sufficient for stability in the dynamic regulation layer. It is therefore of interest to characterize the cases when dissipativity is sufficient for the stability of the closed loop system.

This work focuses on linear system with purely quadratic costs, without definiteness restrictions. Such purely quadratic costs arise, for instance, in ocean wave energy conversion where the objective is to maximize the absorbed power. The power extracted can be modelled as a product of the damping coefficient (constant factor), velocity of the buoy (state, $x(k)$ ) and the active forcing element $(u(k))$ [8-10]. This leads to an indefinite quadratic formulation of the economic objective function. Such indefinite quadratic costs are also encountered in process control where the economic objective of an isothermal continuous stirred-tank reactor is to maximize the production rate (of one of the outputs), modelled as a product of the concentration of the output (state) and the flow rate through the reactor (input) $[6,11,12]$. Another scenario is when there are conflicting objectives, for instance, minimizing the control effort (energy input) of steering an aircraft while trying to maximize the cruise speed (kinetic energy) of the aircraft $[13,14]$. This can be achieved by minimizing a quadratic cost using a negative weighting $(Q<0)$ on the states and positive weighting $(R>0)$ on the inputs, which once again leads to an indefinite cost formulation.

Hence, we seek to establish conditions under which the optimal economic controller in such cases as these is also an asymptotically stabilizing controller for the system. By creating a link between dissipativity, existence of a control Lyapunov function for the closed-loop system and the optimal cost function, we establish a necessary and sufficient condition for stability of the closed-loop system based on dissipativity of the system with respect to the stage cost.

This paper is organized as follows: Section 2 investigates the link between 
dissipativity and the existence of a stabilizing optimal controller; Section 3 presents a discussion on the sufficiency of strict-dissipativity for closed-loop asymptotic stability and possibility of dissipativity to guarantee stability; Section 4 characterizes the necessary and sufficient condition under which dissipativity guarantees asymptotic stability; Section 5 presents some numerical examples and Section 6 concludes the paper.

Nomenclature. The symbols $\mathbb{R}$ and $\mathbb{I}_{0: N-1}$ denote the sets of real numbers and $\{0,1, \ldots . N-1\}$ respectively. We denote $\rho(C)$ as the spectral radius of $\mathrm{C}, C^{\dagger}$ as the Moore-Penrose generalized inverse of $C$ and $\operatorname{Ker}(C)$ as the Kernel of $C$.

\section{On Dissipativity and Existence of Stabilizing Optimal Controller}

In this section, the necessity and sufficiency of dissipativity (of a system with respect to the given objective function) for the optimal controller to be stabilizing is investigated. To ease checking of the dissipativity condition, we focus on linear systems with quadratic cost functions without any restriction on the definiteness of the cost.

Given the linear discrete time system

$$
x_{k+1}=A x_{k}+B u_{k}
$$

and the stage cost

$$
l\left(x_{k}, u_{k}\right)=x_{k}^{T} Q x_{k}+u_{k}^{T} R u_{k}+2 x_{k}^{T} S u_{k},
$$

we consider the following finite-horizon optimization problem

$$
\begin{aligned}
& \min _{\mathbf{u}} J_{N}(x, \mathbf{u}) \triangleq x_{N}^{T} P_{N} x_{N}+\sum_{k=0}^{N-1} l\left(x_{k}, u_{k}\right) \\
& \text { subject to }\left\{\begin{array}{l}
x_{k+1}=A x_{k}+B u_{k}, k \in \mathbb{I}_{0: N-1} \\
x_{k} \in \mathbb{X}, u_{k} \in \mathbb{U}, k \in \mathbb{I}_{0: N-1} \\
x_{0}=x(i), x_{N} \in \mathbb{X}_{F}
\end{array}\right.
\end{aligned}
$$

where $\mathbb{X} \subseteq \mathbb{R}^{n}, \mathbb{U} \subset \mathbb{R}^{m}$ and $\mathbb{X}_{F} \subseteq \mathbb{X}$ is a compact terminal region chosen to ensure recursive feasibility. $x(i)$ is the measured state at time $i$ and $x_{k}$ the predicted value of state $x$ at time step $i+k$ given measurement $x(i)$. Without loss of generality, the optimal steady-state, defined as the solution to the optimization problem

$$
l\left(x_{s}, u_{s}\right)=\min _{x, u} l\left(x_{k}, u_{k}\right) \text { s.t. }\left\{x_{k}=A x_{k}+B u_{k}, x_{k} \in \mathbb{X}, u_{k} \in \mathbb{U}\right\}
$$

is assumed to be the origin, unique and lies in the interior of the constraint sets. Moreover, there is no restriction on the definiteness of the matrix $\left[\begin{array}{cc}Q & S \\ S^{T} & R\end{array}\right]$ and the terminal cost, $P_{N}$. 
The optimization problem (3) is repeatedly minimized over the horizon $N$ in a moving horizon manner. At each iteration $i,(3)$ yields the optimal input sequence $\mathbf{u}^{*}=\left\{u_{0}^{*}, u_{1}^{*}, \ldots, u_{N-1}^{*}\right\}$. The first element of the sequence is applied to the plant yielding the control law $u(i)=u_{0}^{*}$. We refer to this generated implicit control law as $u_{0}^{*}=-K_{N}(x(i))$ and the closed loop system is

$$
x(i+1)=A x(i)-B K_{N}(x(i)) .
$$

If $x_{0}$ is in the set of states that can be admissibly steered to the origin in $N$ steps (or less) and $P_{N}$ is chosen such that it solves the Discrete Algebraic Riccati Equation (DARE)

$$
A^{T} P_{N} A-P_{N}+Q-\left(S+A^{T} P_{N} B\right) K_{N}=0
$$

where $K_{N}=\left(R+B^{T} P_{N} B\right)^{\dagger}\left(S^{T}+B^{T} P_{N} A\right)$ and a solution to (6) is assumed to exist, then the terminal cost is the same as the optimal linear quadratic cost, and hence the cost in (3) is equivalent to an infinite-horizon cost [15-17]. Thus the control law beyond the horizon becomes the linear law $u_{0}^{*}=-K_{N} x(i)$ and stability depends on the stabilising properties of this feedback control law. The closed loop system (4) is thus asymptotically stable if $\rho\left(A-B K_{N}\right)<1$ and marginally stable if $\rho\left(A-B K_{N}\right) \leq 1$ where the eigenvalues with unit modulus have equal algebraic and geometric multiplicity. $P_{N}$ is said to be the stabilizing solution for (DARE) (6) if $P_{N}$ satisfies $(6)$ and $\rho\left(A-B K_{N}\right)<1$. Except where otherwise stated, it is assumed that (6) holds with the terminal cost $P_{N}$ used in $(3)$.

\section{Assumption 2.1.}

- $(A, B)$ is stabilizable.

- $x_{0} \in \mathbb{X}^{0}$ where $\mathbb{X}^{0}$ is the set of states that can be admissibly steered to $\mathbb{X}_{F}$ in $N$ steps (or less).

Definition 1. System (1) is said to be dissipative [4, 18, 19] with respect to the stage cost (2) if there exists a quadratic storage function $x_{k}^{T} P_{d} x_{k}$ such that for all $k \geq 0$,

$$
x_{k+1}^{T} P_{d} x_{k+1}-x_{k}^{T} P_{d} x_{k} \leq l\left(x_{k}, u_{k}\right)
$$

Equation (7) is equivalent to the existence of a $P_{d}=P_{d}^{T}$ such that the dissipativity Linear Matrix Inequality (d-LMI)

$$
\left[\begin{array}{cc}
A^{T} P_{d} A-P_{d}-Q & A^{T} P_{d} B-S \\
B^{T} P_{d} A-S^{T} & B^{T} P_{d} B-R
\end{array}\right] \leq 0
$$

is feasible. If (7) and (8) hold with strict inequality, the system is said to be strictly-dissipative.

We note that while the original definition of dissipativity [20, 21] required $P_{d}$ to be non-negative, recent definitions and usage in economic MPC (e-MPC) have removed this restriction $[4,7,22,23]$. 
Consider the DARE (6). Taking the Schur complement of (6) yields the LMI

$$
\left[\begin{array}{cc}
A^{T} P A-P+Q & A^{T} P B+S \\
B^{T} P A+S^{T} & B^{T} P B+R
\end{array}\right] \geq 0
$$

with $P_{N}$ being the maximum $P$ for which $(9)$ holds. This equivalence was established in [24] where it was shown that

- The set of strongly rank minimizing solutions of the discrete LMI coincide with the set of real symmetric solutions of the DARE associated with the LMI.

- Stabilising and semi-stabilziing rank minimizing solutions of the discrete LMI are also strongly rank minimizing.

- The semi-stabilizing rank minimizing solution of the LMI, if it exists, is the largest solution of the LMI.

We also note that the existence of $P$ that ensures feasibility of $(9)$ is a necessary condition for the existence of $P_{N}$ that solves (6) [17, 24]. Moreover, if (9) is feasible, then $\operatorname{Ker}\left(R+B^{T} P_{N} B\right) \subseteq \operatorname{Ker}\left(A^{T} P_{N} B+S\right)$ which guarantees uniqueness of the generalized inverse in (6).

To begin our investigations, we establish links between dissipativity, existence of solution to the DARE (6) and stabilizing properties of the resulting controller.

Lemma 2.1. $P_{d}$ satisfies (8) if and only if $P=-P_{d}$ satisfies (9).

Proof. The proof is obtained by substituting $P_{d}=-P$ into (8).

Corollary 2.1.1. Existence of $P_{d}$ for which the dissipativity LMI (8) holds is a necessary condition for the closed loop system $x_{k+1}=\left(A-B K_{N}\right) x_{k}$ to be stable.

Proof. This follows from combining Lemma 2.1 with the fact that the existence of a $P$ for which LMI (9) is feasible is a necessary condition for $P_{N}$ to be the stabilizing solution to the DARE (6).

Lemma 2.2. Consider system (1) with two different cost functions (2), $l_{1}$ and $l_{2}$, defined by the quadruples $\left\{Q_{1}, S_{1}, R_{1}, P_{N 1}\right\}$ and $l_{2}=\left\{Q_{2}, S_{2}, R_{2}, P_{N_{2}}\right\}$. Then optimization problem (3) with stage cost $l_{1}$ yields the same controller with stage cost $l_{2}$ if $l_{2}$ is chosen such that $Q_{2}=Q_{1}+P_{d}-A^{T} P_{d} A, S_{2}=S_{1}-A^{T} P_{d} B$, $R_{2}=R_{1}-B^{T} P_{d} B$ and $P_{N_{2}}=P_{N_{1}}+P_{d}$ where $P_{d}$ is such that the $d-L M I$ (8) is feasible for the cost $\left\{Q_{1}, S_{1}, R_{1}\right\}$.

Proof. With the terminal cost in (3) chosen such that $P_{N_{1}}=P_{1}, P_{N_{2}}=P_{2}$ where $P_{1}$ and $P_{2}$ are the solutions to the corresponding DARE, the resulting controllers are equivalent to the corresponding infinite horizon controllers. Hence we can study the behaviours of the controllers by analysing the corresponding DAREs:

$$
A^{T} P_{N 1} A-P_{N 1}-\left(S_{1}+A^{T} P_{N 1} B\right) K_{N 1}+Q_{1}=0
$$




$$
A^{T} P_{N 2} A-P_{N 2}-\left(S_{2}+A^{T} P_{N 2} B\right) K_{N 2}+Q_{2}=0
$$

where $K_{N 1}=\left(R_{1}+B^{T} P_{N 1} B\right)^{\dagger}\left(S_{1}^{T}+B^{T} P_{N 1} A\right)$ and $K_{N 2}=\left(R_{2}+B^{T} P_{N 2} B\right)^{\dagger}\left(S_{2}^{T}+\right.$ $\left.B^{T} P_{N 2} A\right)$. Substituting for $\left\{Q_{2}, S_{2}, R_{2}, P_{N 2}\right\}$ in (11),

$$
\begin{aligned}
& A^{T} P_{N 1} A+A^{T} P_{d} A-P_{1}-P_{d}+Q_{1}+P_{d}-A^{T} P_{d} A \\
& -\left(S_{1}-A^{T} P_{d} B+A^{T} P_{N 1} B+A^{T} P_{d} B\right) \times\left(R_{1}-B^{T} P_{d} B+B^{T} P_{N 1} B+\right. \\
& \left.\quad B^{T} P_{d} B\right)^{\dagger}\left(S_{1}^{T}-B^{T} P_{d} A+B^{T} P_{N 1} A+B^{T} P_{d} A\right)=0
\end{aligned}
$$

Simplifying (12) yields (10) with $K_{N 1}=K_{N 2}$.

Remark 1. As will be used later, Lemma 2.2 is often used to prove stability of the closed loop system in economic MPC setups: if the system is strictlydissipative with respect to the stage cost, the cost can be rotated using $P_{d}$ to get a positive-definite stage cost that yields the same controller as the original cost.

Lemma 2.3. If there exists $P_{d}$ for which the dissipativity LMI (8) is feasible, then for any compatible sized $K_{N}$,

$$
\begin{aligned}
A^{T} P_{d} A-P_{d}-Q- & \left(A^{T} P_{d} B-S\right) K_{N} \\
& -K_{N}^{T}\left(B^{T} P_{d} A-S^{T}\right)+K_{N}^{T}\left(B^{T} P_{d} B-R\right) K_{N} \leq 0 .
\end{aligned}
$$

Proof.

$$
\begin{gathered}
{\left[\begin{array}{rr}
A^{T} P_{d} A-P_{d}-Q & A^{T} P_{d} B-S \\
B^{T} P_{d} A-S^{T} & B^{T} P_{d} B-R
\end{array}\right] \leq 0} \\
\Downarrow \\
{\left[\begin{array}{cc}
I_{n_{x}} & 0 \\
0 & -K_{N}^{T}
\end{array}\right]\left[\begin{array}{cc}
A^{T} P_{d} A-P_{d}-Q & A^{T} P_{d} B-S \\
B^{T} P_{d} A-S^{T} & B^{T} P_{d} B-R
\end{array}\right]\left[\begin{array}{cc}
I_{n_{x}} & 0 \\
0 & -K_{N}
\end{array}\right]} \\
=\left[\begin{array}{cc}
A^{T} P_{d} A-P_{d}-Q & -\left(A^{T} P_{d} B-S\right) K_{N} \\
-K_{N}^{T}\left(B^{T} P_{d} A-S^{T}\right) & K_{N}^{T}\left(B^{T} P_{d} B-R\right) K_{N}
\end{array}\right] \leq 0 \\
\left.\qquad \begin{array}{cc}
A^{T} P_{d} A-P_{d}-Q & -\left(A^{T} P_{d} B-S\right) K_{N} \\
-K_{N}^{T}\left(B^{T} P_{d} A-S^{T}\right) & K_{N}^{T}\left(B^{T} P_{d} B-R\right) K_{N}
\end{array}\right]\left[\begin{array}{l}
I \\
I
\end{array}\right] \\
=A^{T} P_{d} A-P_{d}-Q-\left(A^{T} P_{d} B-S\right) K_{N} \\
-K_{N}^{T}\left(B^{T} P_{d} A-S^{T}\right)+K_{N}^{T}\left(B^{T} P_{d} B-R\right) K_{N} \leq 0 .
\end{gathered}
$$

Lemma 2.4. Let there exist a $P_{d}$ for which the dissipativity $L M I(8)$ is feasible. If $P_{N}$ solves the DARE (6) and $K_{N}$ is chosen such that

$$
K_{N}=\left(R+B^{T} P_{N} B\right)^{\dagger}\left(S^{T}+B^{T} P_{N} A\right),
$$

then

$$
\left(A-B K_{N}\right)^{T}\left(P_{d}+P_{N}\right)\left(A-B K_{N}\right)-\left(P_{d}+P_{N}\right) \leq 0 .
$$


Proof. If $P_{N}$ solves the DARE (6), then (6) can be re-written as

$$
\begin{aligned}
\left(A-B K_{N}\right)^{T} P_{N}\left(A-B K_{N}\right)-P_{N}+Q & \\
& +K_{N}^{T} R K_{N}-S K_{N}-K_{N}^{T} S^{T}=0
\end{aligned}
$$

From Lemma 2.3, we have that the existence of a $P_{d}$ that guarantees feasibility of LMI (8) implies that

$$
\begin{aligned}
A^{T} P_{d} A-P_{d}-Q- & \left(A^{T} P_{d} B-S\right) K_{N} \\
& -K_{N}^{T}\left(B^{T} P_{d} A-S^{T}\right)+K_{N}^{T}\left(B^{T} P_{d} B-R\right) K_{N} \leq 0
\end{aligned}
$$

which can also be re-written as:

$$
\begin{aligned}
\left(A-B K_{N}\right)^{T} P_{d}\left(A-B K_{N}\right)-P_{d}-Q & \\
& -K_{N}^{T} R K_{N}+S K_{N}+K_{N}^{T} S^{T} \leq 0 .
\end{aligned}
$$

Adding (17) and (19) together gives:

$$
\left(A-B K_{N}\right)^{T}\left(P_{d}+P_{N}\right)\left(A-B K_{N}\right)-\left(P_{d}+P_{N}\right) \leq 0
$$

which is what was required.

Lemmata 2.3 and 2.4 attempt to link dissipativity of the system (with respect to the given stage-cost) to the stability of the closed-loop system (5). Inequality (16) mimics a Lyapunov inequality for the closed-loop system. Let $P=P_{d}+P_{N}$. If $\left(P_{d}+P_{N}\right)$ is guaranteed to be positive definite, then (16) is a Lyapunov inequality for marginal stability of the closed-loop system. However, there is no guarantee yet that $\left(P_{d}+P_{N}\right)$ will be positive-definite. Hence it is necessary to characterize conditions under which $\left(P_{d}+P_{N}\right)$ is guaranteed to be positivedefinite.

Remark 2. We note that in the discussions and proofs so far, no restriction has been placed on the definiteness of $P_{d}$ and $P_{N}$. If $P=\left(P_{d}+P_{N}\right)$ is such that $P>0$, we see that dissipativity (hence a storage function $x_{k}^{T} P_{d} x_{k}$ ) gives the link between the optimal cost, $x_{k}^{T} P_{N} x_{k}$, and any Lyapunov function, $x_{k}^{T} P x_{k}$ that proves stability of the closed-loop system. Moreover, it is now clear why there is no need to place a restriction on the definiteness of $P_{d}$.

\section{Sufficiency of Strict-dissipativity}

Before characterizing conditions that guarantee positive-definitess of $\left(P_{d}+\right.$ $P_{N}$ ), we consider the question: is strict-dissipativity really necessary or just sufficient for the closed loop system (5) to be asymptotically stable? This question is motivated by the fact that in e-MPC literature, proof of asymptotic stability of the closed loop system is often based on an assumption that the system is strictly-dissipative with respect to the stage-cost $[4,7,22,23]$. Furthermore in a 
recent paper [25], the authors stated, using the principles of dynamic programming, that dissipativity is not enough to guarantee asymptotic stability of the closed loop system in the case of a general nonlinear system.

Let us consider system (1) where $A=\left[\begin{array}{cc}0 & 1 \\ -2 & -5\end{array}\right]$ and $B=\left[\begin{array}{l}0 \\ 1\end{array}\right]$ with two stage costs described by :

- $l_{1}: Q=0, R=0, S=\left[\begin{array}{l}1 \\ 1\end{array}\right]$

- $l_{2}: Q=0, R=0, S=\left[\begin{array}{l}1 \\ 2\end{array}\right]$

The system is only dissipative with respect to the cost described by $l_{1}$ while it is strictly-dissipative with respect to the cost described by $l_{2}$. Rotating the stage costs with the respective $P_{d} s$ and solving the respective DAREs, the infinite horizon optimal feedback gains are calculated to be $K_{N 1}=\left[\begin{array}{ll}-1.8246 & -3.8246\end{array}\right]$ and $K_{N 2}=\left[\begin{array}{ll}-1.944 & -4.4171\end{array}\right]$ with $\rho\left(A-B K_{N 1}\right)=1$ and $\rho\left(A-B K_{N 2}\right)=$ 0.4617. From Definition 1, let the dissipation residual, $D_{k}$, be defined as

$$
D_{k} \triangleq x_{k+1}^{T} P_{d} x_{k+1}-x_{k}^{T} P_{d} x_{k}-l\left(x_{k}, u_{k}\right) \leq 0
$$

Substituting the infinite horizon optimal controller as $u_{k}$ in (20), we plot the dissipation residuals from an initial condition $x_{0}=[2,1]$ using the respective $P_{d} s$ (with which the stage cost was rotated) in (20) as shown in Figure 1.We note that although both dissipation residuals are non-positive for the respective optimal inputs, the optimal inputs yield different closed-loop behaviours as implied by the spectral properties of the respective closed-loop systems. This confirms that dissipativity alone is not enough to guarantee asymptotic stability (though it may guarantee marginal stability). But then, is strict-dissipativity needed?

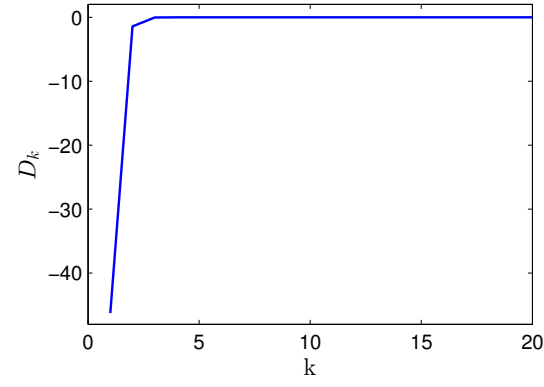

(a)

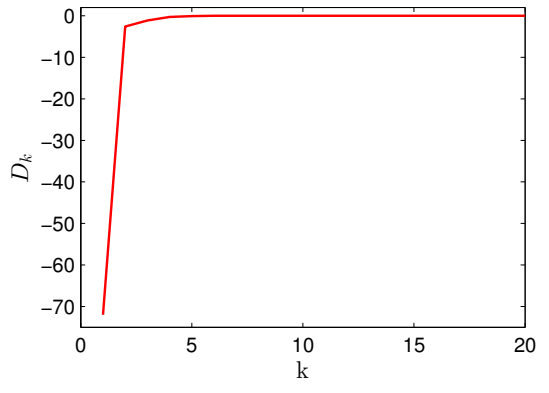

(b)

Figure 1: The dissipation trajectory for the system with $l_{1}(1(\mathrm{a}))$ and $l_{2}(1(\mathrm{~b}))$

In the discussion that follows, we consider the cases when the system is strictly-dissipative and only dissipative with respect to the stage-cost and try to link these properties to the asymptotic stability of the closed-loop system. 
Let us assume that $K_{N}$ has been chosen as in (15) where $P_{N}$ solves the DARE (6). Let us also assume that $\left(P_{d}+P_{N}\right)=P$ in Lemma 2.4 is positive-definite and that the closed loop system is at least marginally stable i.e

$$
\left(A-B K_{N}\right)^{T} P\left(A-B K_{N}\right)-P \leq 0 .
$$

Combining (17) with (21) and rearranging yields

$$
\begin{aligned}
A^{T} P_{d} A-P_{d}-Q- & \left(A^{T} P_{d} B-S\right) K_{N} \\
& -K_{N}^{T}\left(B^{T} P_{d} A-S^{T}\right)+K_{N}^{T}\left(B^{T} P_{d} B-R\right) K_{N} \leq 0 .
\end{aligned}
$$

Hence marginal stability of the closed loop system is equivalent to (22). If (21) (hence (22)) holds with strict inequality, the closed-loop system is asymptotically stable.

\subsection{Case 1: System strictly-dissipative with respect to the stage-cost}

For a system that is strictly-dissipative with respect to the stage-cost, we have from Definition 1 that there exists $P_{d}$ such that the LMI

$$
\left[\begin{array}{cc}
A^{T} P_{d} A-P_{d}-Q & A^{T} P_{d} B-S \\
B^{T} P_{d} A-S^{T} & B^{T} P_{d} B-R
\end{array}\right]<0 .
$$

Given $K_{N}$ and applying Lemma 2.3, we know that (23) implies that

$$
\begin{array}{r}
\underbrace{\left[\begin{array}{ll}
I & -K_{N}^{T}
\end{array}\right]}_{X^{T}} \underbrace{\left[\begin{array}{rr}
A^{T} P_{d} A-P_{d}-Q & A^{T} P_{d} B-S \\
B^{T} P_{d} A-S^{T} & B^{T} P_{d} B-R
\end{array}\right]}_{\Downarrow} \underbrace{\left[\begin{array}{c}
I \\
-K_{N}
\end{array}\right]}_{\mathrm{Y}} \leq 0 \\
A^{T} P_{d} A-P_{d}-Q-\left(A^{T} P_{d} B-S\right) K_{N} \\
-K_{N}^{T}\left(B^{T} P_{d} A-S^{T}\right)+K_{N}^{T}\left(B^{T} P_{d} B-R\right) K_{N} \leq 0 .
\end{array}
$$

Since $Y<0$ by strict-dissipativity, the rank of (24) is equal to the rank of $X$, which by construction has full column rank. Thus, (24) holds with strict inequality. From (22), asymptotic stability of the closed-loop system is equivalent to (24) holding. This implies that by strict-dissipativity, the closed loop system is guaranteed to be asymptotically stable.

However, strict-dissipativity implies that for all $x_{k}, u_{k}$ (including the optimal pair), (7) holds with strict inequality. Invoking Lemma 2.1 and substituting into (23) implies that

$$
\left[\begin{array}{cc}
A^{T} P A-P+Q & A^{T} P B+S \\
B^{T} P A+S^{T} & B^{T} P B+R
\end{array}\right]>0
$$

$P_{N}$ (which corresponds to the maximum $P$ ) is usually such that $(25)$ is positive semi-definite. Hence we see that requiring LMI (25) to hold with strict inequality is quite a strong condition to be placed on the system and is not really necessary (since $(25)$ does not hold with $P_{N}$ ) but it is sufficient to guarantee asymptotic stability of the closed-loop system. 
3.2. Case 2: System dissipative with respect to the stage-cost

In the case of a system that is only dissipative with respect to the stagecost, (23) holds with non-strict inequality. Given $K_{N}$ and applying Lemma 2.3 implies that

$$
\underbrace{\left[\begin{array}{cc}
I & -K_{N}^{T}
\end{array}\right]}_{X_{1}^{T}} \underbrace{\left[\begin{array}{cc}
A^{T} P_{d} A-P_{d}-Q & A^{T} P_{d} B-S \\
B^{T} P_{d} A-S^{T} & B^{T} P_{d} B-R
\end{array}\right]}_{Y_{1}} \underbrace{\left[\begin{array}{c}
I \\
-K_{N}
\end{array}\right]}_{X_{1}} \leq 0
$$

with (24) holding as before. Hence, dissipativity guarantees marginal stability of the closed-loop system (provided $\left(P_{d}+P_{N}\right)>0$ ). Marginal stability is however not usually enough. Is there any possibility of asymptotic stability i.e (24) being negative-definite when $Y_{1}$ is not full rank? We know from Linear Algebra that this is possible. For example, given an arbitrary $K_{N}=\left[\begin{array}{ll}1 & 2 \\ 3 & 4\end{array}\right]$ and arbitrary $Y_{1}=\left[\begin{array}{cccc}-1 & 0 & 0 & 0 \\ 0 & -1 & 0 & 0 \\ 0 & 0 & -1 & 0 \\ 0 & 0 & 0 & 0\end{array}\right], X_{1}^{T} Y_{1} X_{1}<0$ although $Y_{1} \leq 0$. This shows that dissipativity can guarantee asymptotic stability.

\section{On the Sufficiency of Dissipativity}

From Section 3, we know that asymptotic stability of the closed loop system when the system is only dissipative with respect to the stage-cost is possible. In this section, we derive conditions that guarantee the stability of the closed-loop system (5) when the optimal feedback gain $K_{N}$ is used. The main assumption here is that the system is dissipative with respect to the stage-cost.

Definition 2. Given $\{Q, S, R\}, \mathcal{P}_{d}$ is defined as the set of all $P_{d}$ for which (8) holds i.e

$$
\mathcal{P}_{d} \triangleq\left\{P_{d} \mid \quad\left[\begin{array}{cc}
A^{T} P_{d} A-P_{d}-Q & A^{T} P_{d} B-S \\
B^{T} P_{d} A-S^{T} & B^{T} P_{d} B-R
\end{array}\right] \leq 0\right\}
$$

Definition 3. $\mathcal{P}_{d}^{o}$ is defined as the set of all $P_{d} \in \mathcal{P}_{d}$ for which there exists at least two distinct $P_{d} s, P_{d}^{i}$ and $P_{d}^{j}$, such that $P_{d}^{i}-P_{d}^{j}$ is of full rank i.e

$$
\mathcal{P}_{d}^{o} \triangleq\left\{P_{d} \in \mathcal{P}_{d} \mid \exists P_{d}^{i} \text { and } P_{d}^{j} \text { such that } P_{d}^{i}-P_{d}^{j} \text { is full rank }\right\}
$$

Lemma 4.1. If $\mathcal{P}_{d}^{o}$ is not empty, then $K_{N}$ is an asymptotically stabilizing feedback gain i.e $\rho\left(A-B K_{N}\right)<1$.

Proof. Consider the dissipativity LMI (8). Provided there exists a $P_{d}$ such that (8) is feasible (irrespective of the definiteness of $\left[\begin{array}{cc}Q & S \\ S^{T} & R\end{array}\right]$ ), the 'rotated' stage $\operatorname{cost}\left[\begin{array}{cc}\hat{Q} & \hat{S} \\ \hat{S}^{T} & \hat{R}\end{array}\right]$ is positive semi-definite where

$$
\begin{aligned}
& \hat{Q}=Q+P_{d}-A^{T} P_{d} A \\
& \hat{S}=S-A^{T} P_{d} B \\
& \hat{R}=R-B^{T} P_{d} B
\end{aligned}
$$


Lemma (2.2) shows that the original cost and rotated cost (28) yield the same controller hence we can study the properties of the controller obtained using the rotated cost instead of the original cost. Consider the DARE:

$$
A^{T} \hat{P} A-\hat{P}-\left(\hat{S}+A^{T} \hat{P} B\right)\left(\hat{R}+B^{T} \hat{P} B\right)^{\dagger}\left(\hat{S}^{T}+B^{T} \hat{P} A\right)+\hat{Q}=0
$$

By positive semi-definiteness of the rotated cost, we are guaranteed that $\hat{P} \geq 0$ solves (29). Now consider dissipativity of the system with respect to the rotated stage cost (28) expressed as

$$
\left[\begin{array}{cc}
A^{T} P_{\delta} A-P_{\delta}-\hat{Q} & A^{T} P_{\delta} B-\hat{S} \\
B^{T} P_{\delta} A-\hat{S}^{T} & B^{T} P_{\delta} B-\hat{R}
\end{array}\right] \leq 0
$$

Since the rotated cost is positive semi-definite, we are assured of at least one $P_{\delta}$ for which (30) is feasible, namely $P_{\delta}=0$.

If the closed loop system (5) is asymptotically stable with $K_{N}=(\hat{R}+$ $\left.B^{T} \hat{P} B\right)^{\dagger}\left(\hat{S}^{T}+B^{T} \hat{P} A\right)$, we are able to find a $P>0$ such that

$$
\left(A-B K_{N}\right)^{T} P\left(A-B K_{N}\right)-P<0 .
$$

Now let $P=P_{\delta}+\hat{P}$ as in Lemma 2.4. If we can find $P_{\delta}>0$ that ensures feasibility of (30), then we are guaranteed that $P>0$ (since $\hat{P} \geq 0$ ). While it suffices that $P_{\delta}>0$, we note that it is not a necessary condition as long as $P_{\delta}$ is of full rank and ensures feasibility of (30). This is because if $K_{N}$ is such that the closed-loop system is asymptotically stable, the fact that $P>0$ exists that satisfies (31) does not imply that $P-\hat{P}$ is always positive-definite. Moreover, we can apply Lemma 2.1 to show that $P_{\delta}+\hat{P}$ is always non-negative. Hence a sufficient condition to guarantee the stability of the closed loop system is the existence of a full rank $P_{\delta}$ for which the dissipativity LMI (30) holds.

Substituting (28) into (30), the dissipativity condition (30) can be expressed in terms of the original stage cost as

$$
\left[\begin{array}{cc}
A^{T} P_{\delta} A-P_{\delta}-Q-P_{d}+A^{T} P_{d} A & A^{T} P_{\delta} B-S+A^{T} P_{d} B \\
B^{T} P_{\delta} A-S^{T}+B^{T} P_{d} A & B^{T} P_{\delta} B-R+B^{T} P_{d} B
\end{array}\right] \leq 0
$$

Let $P_{\theta}=P_{\delta}+P_{d},(32)$ then becomes

$$
\left[\begin{array}{cc}
A^{T} P_{\theta} A-P_{\theta}-Q & A^{T} P_{\theta} B-S \\
B^{T} P_{\theta} A-S^{T} & B^{T} P_{\theta} B-R
\end{array}\right] \leq 0
$$

Equation (33) is a dissipativity condition on the system with respect to the original stage cost. Hence, existence of $P_{\theta}$ and $P_{d}$, both elements of $\mathcal{P}_{d}$ such that $P_{\theta}-P_{d}$ is of full rank guarantees the asymptotic stability of the closed loop system (5).

From (22) in section 3, asymptotic stability of the closed-loop system is equivalent to the existence of $P_{d}$ such that

$$
\begin{aligned}
A^{T} P_{d} A-P_{d}-Q- & \left(A^{T} P_{d} B-S\right) K_{N} \\
& -K_{N}^{T}\left(B^{T} P_{d} A-S^{T}\right)+K_{N}^{T}\left(B^{T} P_{d} B-R\right) K_{N}<0
\end{aligned}
$$


Equation (34) is actually the strict-dissipativity inequality from Definition 1 however with $u_{k}=-K_{N} x_{k}$. So given a system that is only dissipative with respect to the given cost, if the infinite horizon optimal input will be such that the closed-loop system is asymptotically stable, then the system must be strictlydissipative (with respect to the cost) when the optimal control law is chosen as $u_{k}=-K_{N} x_{k}$. So we see that though strict-dissipativity is required, it is only needed in a 'local' and not a global sense as in Definition 1. It is needed only with the optimal control law. And what Lemma 4.1 has done is to give the condition that guarantees this 'local' strict-dissipativity condition will hold. A system that is strictly-dissipative with respect to the stage cost automatically fulfils this condition since strict-dissipativity holds for all $u_{k}$, including the optimal control law.

Remark 3. The result shown here is for a quadratic cost (2) with no definiteness restriction. In the case of a positive semi-definite cost (2), it suffices to find only $P_{\theta}$ of full rank. This is because $P_{d}=0$ is feasible if the cost function is positive semi-definite. Hence, if $P_{\theta}$ is full rank, then there exist a $P_{\delta}$ that is of full rank.

We are now ready to state the main result of this paper.

Theorem 4.2. Consider optimization problem (3) and the resulting closed loop system (5). Let Assumption 2.1 hold and the terminal cost $P_{N}$ in (3) be chosen such that $P_{N}$ solves (6). Then the closed loop system (5) is asymptotically stable if and only if the set $\mathcal{P}_{d}^{o}$ is not empty, with the region of attraction $\mathbb{X}^{0}$.

Proof.

If $x_{0} \in \mathbb{X}^{0}$, then there exists $k \leq N$ such that $x_{k} \in \mathbb{X}_{\infty}$ where $\mathbb{X}_{\infty}$ is the maximal admissible set for the closed-loop system $\left(A-B K_{N}\right) x$. With this choice of $x_{0}$, the solutions to the constrained optimization problem (3) and the unconstrained infinite horizon regulator problem coincide [15] and stability of the closed-loop system can be analysed based on the stabilizing properties of the terminal controller $u=-K_{N} x_{k}$ where $K_{N}=\left(\hat{R}+B^{T} \hat{P} B\right)^{\dagger}\left(\hat{S}^{T}+B^{T} \hat{P} A\right)$. Hence we consider stabilizing properties of this controller.

Sufficiency. Lemma 4.1 shows the sufficiency of $\mathcal{P}_{d}^{o}$ not being an empty set for the optimal feedback gain $K_{N}$ to be locally asymptotically stabilizing.

Necessity. Lemma 2.1 shows the necessity of dissipativity and sufficiency of dissipativity for the existence of $P_{N}$ that solves (6). From Lemma 2.4, for the system to be stable, there must exist a $P_{d}$ such that

$$
P_{d}>-P_{N} .
$$

Applying Lemma 2.1, $P_{d}=-P \Longrightarrow-P_{N}=\min \left(P_{d}\right)$. Hence, (35) cannot hold if the set $\mathcal{P}_{d}^{o}$ is empty.

Corollary 4.2.1. Let $K$ be a stabilizing feedback gain for a system such that $\rho(A-B K)<1$. Then there is at least one $\left\{Q, S, R, P_{d}\right\}$ quadruple for which the system is strictly-dissipative i.e (34) holds with $K_{N}=K$. Furthermore, if no such quadruple exists, then $K$ is not a stabilizing feedback gain. 
Remark 4. The minimum cardinality of $\mathcal{P}_{d}^{o}$ is 2. A necessary condition for the existence of $\mathcal{P}_{d}^{o}$ is that $\mathcal{P}_{d}$ is not a singleton. Hence, in situations where there exists only one $P_{d}$ for which (8) holds, the resulting controller will not be asymptotically stabilizing. An example of this is in systems where the 'available' and 'required' storage functions [21] are equal. Since all storage functions for the system in this context are upper bounded by the required storage and lower bounded by the available storage, equivalence of these two functions implies there is only one storage function for which the system is dissipative with respect to the given cost, hence only one $P_{d}$ that satisfies the dissipativity LMI (8).

Remark 5. For a system with $\mathcal{P}_{d}^{o}$ being an empty set, only the infinite horizon (and its finite-horizon equivalent when the terminal cost is chosen to be the solution to the DARE) has been shown not to be stabilizing. The system can still be stabilized by using a stabilizing terminal cost.

\section{Algorithm for finding the Stabilizing Controller}

Given optimization problem (3) where $P_{N}$ has been chosen such that it solves (6), we describe an algorithm for checking if $\mathcal{P}_{d}^{o}$ is not empty and finding the stabilizing feedback gain, $K_{N}$ (if it exists).

The set of all storage functions for which system (1) is dissipative with respect to the stage cost (2) is known to be convex [21, 26]. Moreover, $P_{d}^{l} \leq$ $P_{d} \leq P_{d}^{u}$ where $P_{d}^{l}$ is the minimum $P_{d}$ for which LMI (8) is feasible and $P_{d}^{u}$ the maximum $P_{d}$ for which (8) is feasible. It follows that $\mathcal{P}_{d}$ is partially ordered.

Thus, existence of $\left(P_{\theta}-P_{d}\right)=P_{\delta}$ of full rank is equivalent to $P_{d}^{u}-P_{d}^{l}$ being of full rank.

\section{Algorithm 4.3.}

Given $\{A, B, Q, R, S\}$

1. Set up a semi-definite programming (SDP) problem to find the minimum $P_{d}$ for which LMI (8) is feasible. This is $P_{d}^{l}$.

2. Set up another SDP problem to find the maximum $P_{d}$ for which LMI (8) is feasible. This is $P_{d}^{u}$.

3. If $P_{d}^{u}-P_{d}^{l}$ has full rank, then $P_{\delta}$ of full rank exists, $\mathcal{P}_{d}^{o}$ is not empty and $K_{N}$ is asymptotically stabilizing. Else, $K_{N}$ is not asymptotically stabilizing.

To compute the asymptotically stabilizing feedback gain,

4. Using any $P_{d}$ for which $L M I(8)$ is feasible, rotate the original cost such that $\hat{Q}=Q+P_{d}-A^{T} P_{d} A, \hat{S}=S-A^{T} P_{d} B, \hat{R}=R-B^{T} P_{d} B$.

5. Set up another $S D P$ problem to find the maximum $P$ (by maximizing the determinant of $P$ or any other method) such that LMI (8) is feasible.

$$
\left[\begin{array}{cc}
A^{T} P A-P+\hat{Q} & A^{T} P B+\hat{S} \\
B^{T} P A+\hat{S}^{T} & B^{T} P B+\hat{R}
\end{array}\right] \geq 0 .
$$

6. Obtain the solution to DARE (6) as $P_{N}=P-P_{d}$. Then the asymptotically stabilizing feedback gain is $K_{N}=\left(B^{T} P_{N} B+R\right)^{\dagger}\left(B^{T} P_{N} A+S^{T}\right)$. 


\section{Numerical Examples}

This section contains examples to show the results of Theorem 4.2 using Algorithm 4.3. In all examples shown, the system considered is only dissipative with respect to the cost function given.

\subsection{Example 1}

We consider system (1) and stage cost (2) where

$$
A=\left[\begin{array}{cc}
1.15 & 0 \\
0.1 & 1.1
\end{array}\right], B=\left[\begin{array}{cc}
1 & -1 \\
0 & 0
\end{array}\right], Q=R=0, S=\left[\begin{array}{cc}
-1 & 1 \\
1 & -1
\end{array}\right]
$$

Running Algorithm 4.3, we find $P_{d}=\left[\begin{array}{cc}-1.3415 & 0.6931 \\ 0.6931 & -4.6804\end{array}\right]$ with $P_{\delta}=\left[\begin{array}{cc}1.3232 & 1.7753 \\ 1.7753 & 11.2689\end{array}\right]$ of full rank which implies $\mathcal{P}_{d}^{o}$ is not empty. The infinite horizon optimal feedback gain, $K_{N}=\left[\begin{array}{cc}0.3569 & 0.5536 \\ -0.3569 & -0.5536\end{array}\right]$ and $\rho\left(A-B K_{N}\right)=0.7685$.

\subsection{Example 2}

Given

$$
\begin{gathered}
A=\left[\begin{array}{cr}
1 & 2 \\
-1 & 2
\end{array}\right], B=\left[\begin{array}{cccc}
1 & 0 & -1 & 0 \\
0 & -1 & 0 & 1
\end{array}\right], Q=0, R=0, \\
S=\left[\begin{array}{cccc}
-0.58 & -0.66 & 0.58 & 0.66 \\
-0.39 & 0.56 & 0.39 & -0.56
\end{array}\right] .
\end{gathered}
$$

The system is only dissipative with respect to the stage cost. From Algorithm 4.3, $P_{d}=\left[\begin{array}{cc}-1.5113 & 0.3684 \\ 0.3684 & -1.3537\end{array}\right]$ for which LMI (8) is feasible and $P_{\delta}=$ $\left[\begin{array}{ll}-0.9158 & -0.1274 \\ -0.1274 & -2.8596\end{array}\right]$ has full rank. The infinite horizon optimal feedback gain is computed as $K_{N}=\left[\begin{array}{cc}0.3823 & 0.9 \\ 0.4197 & -0.9136 \\ -0.3823 & -0.9 \\ -0.4197 & 0.9136\end{array}\right]$ with $\rho\left(A-B K_{N}\right)=0.2698$.

\subsection{Example 3}

In this example, we revisit the system from section 3 where

$$
A=\left[\begin{array}{cc}
0 & 1 \\
-2 & -5
\end{array}\right], B=\left[\begin{array}{l}
0 \\
1
\end{array}\right], Q=0, R=0, S=\left[\begin{array}{l}
1 \\
1
\end{array}\right] .
$$

As shown earlier in section 3, the closed-loop system is only marginally stable when the infinite horizon optimal control input is applied. Running Algorithm 4.3 , though $P_{d}=\left[\begin{array}{ll}-0.9916 & -2.9916 \\ -2.9916 & -2.9916\end{array}\right]$ exists for which the system is dissipative with respect to the stage cost, we cannot find $P_{\delta}$ of full rank. This explains why the closed-loop system is not asymptotically stable. 
However, as stated in Remark 5 when the rank condition on $P_{\delta}$ is not satisfied, only the infinite horizon (and the finite horizon equivalent when the solution to the DARE (6) is chosen as the terminal cost) optimization problem has been shown not to result in an asymptotically stable closed-loop system. We can regain asymptotic stability by choosing a stabilizing terminal cost.

Using a terminal cost $P_{N}=\left[\begin{array}{ll}1 & 0 \\ 0 & 1\end{array}\right]$ in optimization problem (3) with an initial condition $x_{0}=[2,1]$, the resulting closed-loop system is asymptotically stable as shown in Figure 2. The horizon length used was $N=20$.
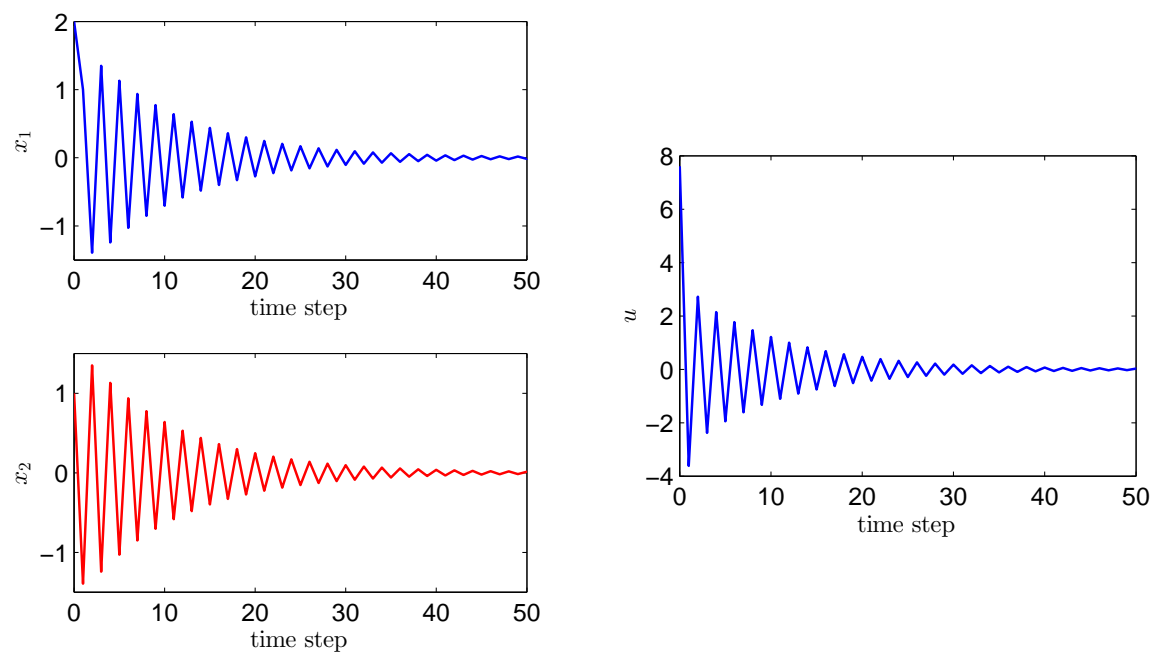

Figure 2: Closed-loop trajectories of the system with a stabilizing terminal cost

\section{Conclusion}

Strict-dissipativity of a system with respect to the objective function has been known to guarantee closed-loop stability when an optimal control in the economic MPC context is used. In this paper we showed that while strictdissipativity guarantees stability of the closed-loop system, it is not really needed when dealing with linear systems with purely quadratic stage costs. We then established a necessary and sufficient condition that guarantees asymptotic stability of the closed-loop system based on dissipativity of the linear system with the quadratic stage cost. This condition is equivalent to the system being strictlydissipative with respect to the objective function when the input is chosen to be the optimal controller. The result comes down to the existence of two distinct storage functions for which the system is dissipative with respect to the cost function. In this paper we restricted ourselves to linear systems with purely quadratic costs; this allows dissipativity to be tested for using the LMI frame- 
work. Extension to costs including linear terms and more general settings will be the focus of future research.

\section{Acknowledgements}

This research is funded by the Federal Government of Nigeria through the Presidential Special Scholarship Scheme for Innovation and Development.

\section{References}

[1] R. R. Bitmead, M. Gevers, V. Wertz, Adaptive Optimal Control: The Thinking Man's G.P.C., Prentice Hall, 1991.

[2] E. Mosca, Optimal, Predictive, and Adaptive Control, Prentice Hall, 1995.

[3] J. Maciejowski, Predictive Control with Constraints, Prentice Hall, 2002.

[4] D. Angeli, R. Amrit, J. Rawlings, On average performance and stability of economic model predictive control, IEEE Transactions on Automatic Control, 57 (7) (2012) 1615-1626. doi:10.1109/TAC.2011.2179349.

[5] M. A. Múller, D. Angeli, F. Allgówer, On necessity and robustness of dissipativity in economic model predictive control, IEEE Transactions on Automatic Control, 60 (6) (2015) 1671-1676.

[6] T. Faulwasser, M. Korda, C. Jones, D. Bonvin, Turnpike and dissipativity properties in dynamic real-time optimization and economic MPC, in: 53rd IEEE Conference on Decision and Control, 2014, pp. 2734-2739.

[7] R. Amrit, J. B. Rawlings, D. Angeli, Economic optimization using model predictive control with a terminal cost, Annual Reviews in Control 35 (2) (2011) 178 - 186. doi :http://dx.doi.org/10.1016/j.arcontrol. 2011. 10.011.

[8] E. Abraham, E. C. Kerrigan, Optimal active control and optimization of a wave energy converter, IEEE Transactions on Sustainable Energy 4 (2) (2013) 324-332.

[9] J. A. Cretel, G. Lightbody, G. P. Thomas, A. W. Lewis, Maximisation of energy capture by a wave-energy point absorber using model predictive control, Proceedings of the 18th IFAC World Congress, Milano, Italy (2011) $3714-3721$.

[10] G. Li, M. R. Belmont, Model predictive control of sea wave energy converters - part i: A convex approach for the case of a single device, Renewable Energy 69 (2014) $453-463$.

[11] T. Faulwasser, D. Bonvin, On the design of economic NMPC based on approximate turnpike properties, in: 54th IEEE Conference on Decision and Control, 2015, pp. $4964-4970$. 
[12] R. Rothfuss, J. Rudolph, M. Zeitz, Flatness based control of a nonlinear chemical reactor model, Automatica 32 (10) (1996) 1433-1439.

[13] R. R. Levary, Optimal control problems with goal objective functions, International Journal of Systems Science 17 (1) (1986) 97-109.

[14] R. R. Levary, Optimal control problems with multiple goal objectives, Optimal Control Applications and Methods 7 (2) (1986) 201-207.

[15] D. Chmielewski, V. Manousiouthakis, On constrained infinite-time linear quadratic optimal control, Systems \& Control Letters 29 (3) (1996) 121 129. doi:http://dx.doi.org/10.1016/S0167-6911(96)00057-6.

[16] A. Bemporad, M. Morari, V. Dua, E. N. Pistikopoulos, The explicit linear quadratic regulator for constrained systems, Automatica 38 (1) (2002) 3 20. doi:http://dx.doi.org/10.1016/S0005-1098(01)00174-1.

[17] M. Zanon, S. Gros, M. Diehl, Indefinite linear MPC and approximated economic MPC for nonlinear systems, Journal of Process Control 24 (8) (2014) 1273 - 1281, economic nonlinear model predictive control. doi: http://dx.doi.org/10.1016/j.jprocont.2014.04.023.

[18] G. C. Goodwin, K. S. Sin, Adaptive Filtering Prediction and Control, Englewood Cliffs, New Jersey 07632: Prentice-Hall, Inc., 1984.

[19] N. Kottenstette, P. J. Antsaklis, Relationships between positive real, passive dissipative, \& positive systems, in: American Control Conference (ACC), 2010, IEEE, 2010, pp. 409-416.

[20] J. Willems, Dissipative dynamical systems part ii: Linear systems with quadratic supply rates, Archive for Rational Mechanics and Analysis 45 (5) (1972) 352-393. doi:10.1007/BF00276494.

[21] J. C. Willems, Dissipative dynamical systems, European Journal of Control 13 (2007) 134-151. doi:http://dx.doi.org/10.3166/ejc.13.134-151.

[22] M. Diehl, R. Amrit, J. Rawlings, A lyapunov function for economic optimizing model predictive control, IEEE Transactions on Automatic Control, 56 (3) (2011) 703-707. doi:10.1109/TAC.2010.2101291.

[23] J. Rawlings, D. Angeli, C. Bates, Fundamentals of economic model predictive control, in: Decision and Control (CDC), 2012 IEEE 51st Annual Conference on, 2012, pp. 3851-3861. doi:10.1109/CDC.2012.6425822.

[24] A. A. Stoorvogel, A. Saberi, The discrete algebraic riccati equation and linear matrix inequality, Linear Algebra and its Applications 274 (1-3) (1998) 317 - 365. doi:http://dx.doi.org/10.1016/S0024-3795 (97)00365-0.

[25] M. Zanon, S. Gros, M. Diehl, Local properties of economic NMPC, dissipativity and dynamic programming, in: Decision and Control (CDC), 2014 IEEE 53rd Annual Conference on, 2014, pp. 2746-2751. 
[26] C. Scherer, S. Weiland, Linear matrix inequalities in control, Lecture Notes, Dutch Institute for Systems and Control, Delft, The Netherlands. 